Dennis J. Ternet

Ronald G. Larson

L. Gary Leal

\section{Transient director patterns upon flow start-up of nematic liquid crystals (an explanation for stress oscillation damping)}

Received: 11 August 2000

Accepted: 31 October 2000

D. J. Ternet

Department of Chemical Engineering

Materials Science and Engineering

Research Center, University of California

at Santa Barbara

Santa Barbara, CA 93106, USA

R. G. Larson ( $\square)$

Department of Chemical Engineering

University of Michigan

Ann Arbor, MI 48109, USA

e-mail: rlarson@engin.umich.edu

L. G. Leal

Departments of Chemical and Materials

Engineering, Materials Science and Engineering Research Center

University of California at Santa Barbara

Santa Barbara, CA 93106, USA

\begin{abstract}
In this work we attempt to determine the origin of damped stress oscillations upon flow start-up of a nematic liquid crystalline monodomain. These damped stress oscillations were first observed by $\mathrm{Gu}$ et al. (1993) in the cone-plate flow cell and have since also been observed by Mather et al. (1997) in the parallel disk cell. Although Mather's work explained the cause of the stress oscillation damping in the torsional flow cell, the origin of the damping in the cone-plate device remains a mystery. Here we report finding similar damped stress oscillations in the cylindrical Couette cell and combined with the optical experiments reported earlier by
\end{abstract}

Cladis and Torza $(1975,1976)$ we are able to propose an explanation for the damping in this geometry. We also report new optical experiments using the cone-plate cell in hopes of determining a cause to the damping in the cone-plate cell.

Key words Liquid crystal . Instability

\section{Introduction}

The first experimental observation of stress oscillations upon shear start-up of a small-molecule tumbling nematic liquid crystal was made by $\mathrm{Gu}$ et al. (1993; $\mathrm{Gu}$ and Jamieson 1994). In their work with a cone-plate flow cell, and the tumbling nematic $8 \mathrm{CB}$, they observed stress oscillations which dampen with increasing strain for all shear-rates and at all temperatures at which $8 \mathrm{CB}$ is known to tumble. In addition to the damping, a peculiar aspect of these experiments was that a nearly perfect mirror image of the damped stress oscillations could be achieved if the flow were reversed at short strains $(\gamma \approx 200)$, while a complete lack of reversibility was found at longer strains. An example of the stress oscillations as well as the reversibility phenomena may be seen in Fig. 1.
The existence of stress oscillations is clearly a signature of tumbling beginning from a state of uniform alignment, and the dynamics of this tumbling process can be modeled using Ericksen's transversely isotropic fluid (TIF) model (Ericksen 1960). Upon flow start-up, the TIF model predicts that the tumbling nematic continuously rotates in the flow leading to a periodic shear stress, but without damping. The strain period of these oscillations is related to the tumbling parameter of the nematic, $\lambda=\sqrt{1-\left(2 \pi / \gamma_{p}\right)}$. Adopting this rather simplified version (2D, no elasticity) of the full LeslieEricksen (LE) theory has proven to be an accurate means of measuring the tumbling parameter of nematic LCs (Ternet et al. 1999).

The damping of the stress oscillations in the coneplate cell, however, is not well understood. Gu and 
Fig. 1 Example of stress oscillation damping observed in the cone-plate flow cell with the tumbling nematic 8CB. Top graph shows reversible damping when shearing is reversed at short strains $(\gamma<200$ strain units) while bottom graph shows irreversible damping when shearing is reversed at longer strains $(\gamma>200$ strain units $)$
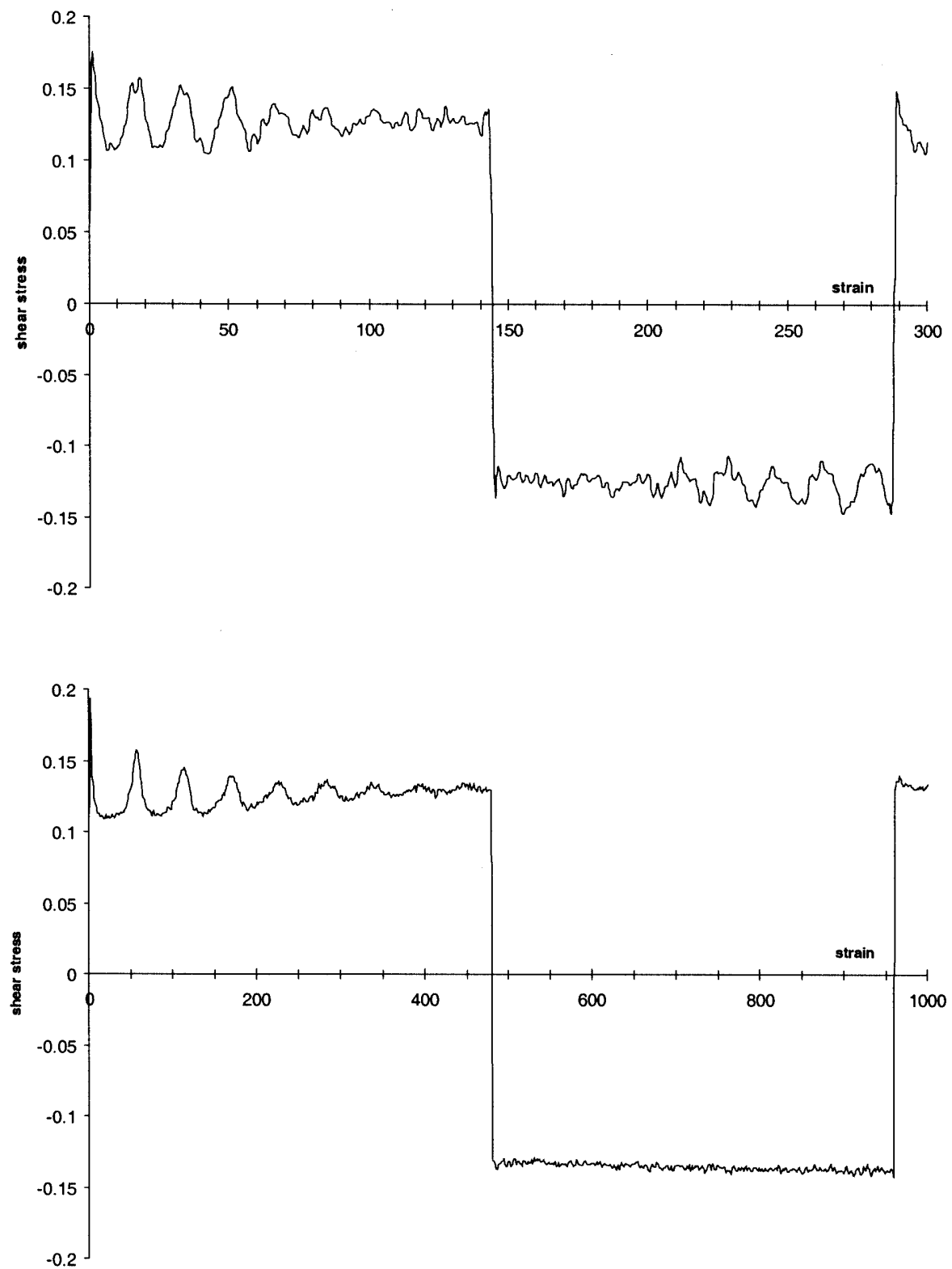

Jamieson (1994) proposed two possible explanations. In the first, it was suggested that the $2 \mathrm{D}$ assumption (i.e., the director remaining in the shear plane) is invalid, and the director simply rotates further and further out of the shear plane at increasing strains. As the director rotates further into the vorticity direction and out of the shear plane, the stress oscillations dampen completely. In the second proposed explanation, the director remains within the shear plane, but the assumption of negligible elasticity is invalid. With the director confined to the shear plane, elastic torques build up due to the continued winding-up of the director from the top plate to the bottom plate. These elastic torques effectively slow down the rotation of the director and produce the damping. Although the increased elasticity proposed by the second explanation would cause the stress oscillations to dampen, it would also cause the strain period to increase at higher strains and the overall stress to increase gradually as well (Burghardt and Fuller 1990), in disagreement with the experimental observations. Neither explanation is capable of explaining the shift from reversible to irreversible damping at higher strains.

The degree of elasticity may be characterized by a dimensionless parameter called the Ericksen number, $E r=\eta \dot{\gamma} /\left(K / h^{2}\right)$, relating the viscous torques to the elastic torques on the director, where $\eta$ is the relevant Leslie viscosity, $\dot{\gamma}$ is the shear rate, $K$ is the relevant Frank elastic constant, and $h$ is the gap. The works of 
Gu et al. (1993; Gu and Jamieson 1994) as well as that of Ternet et al. (1999) had hoped to minimize elastic effects in the measurements by running at very large Ericksen numbers. With typical values for the viscosity $\left(\gamma_{1}\right)$ and elastic constants $\left(K_{3}\right)$ for 8CB (Kneppe et al. 1982; Karat and Madhusudana 1976), the Ericksen numbers used were at least $10^{5}$ at the edge of the cone so it would be surprising if elasticity played a role in the explanation at such an early stage in the shearing.

Mather et al. (1997) approached the problem of trying to explain $\mathrm{Gu}$ and Jamieson's observations in the cone and plate system by examining the flow of the tumbling nematic $8 \mathrm{CB}$ in the parallel disk geometry. In this flow cell they also observed damped stress oscillations although with much faster damping than in the cone-plate cell. It should be noted, however, that in this geometry there is one additional mechanism for damping, and that is the phase mixing due to the radial gradient of shear-rate within the cell. With a nonuniform shear-rate, the director rotation rate is different at different radial positions within the cell, leading to phase mixing which quickly dampens the oscillatory signal. This mechanism can be simulated using Ericksen's TIF model (2D, director assumed to remain in shear plane). However, Mather et al. found that, even after including the phase mixing, the experimental shear stress dampens significantly faster than predicted by the TIF model.

In a second complementary experiment, using polarizing optical microscopy, Mather et al. (1997) observed that the director remains within the plane of shear throughout most of the sample, except in regions called "twist walls" where the director rotates out of the shear plane into the vorticity direction and then back into the shear plane. These twist walls conveniently served as markers of positions within the cell where the director has reached an orientation parallel to the flow direction. Hence, by tracking the rate of radial propagation of these twist walls, Mather and coworkers were able to infer the rate of director rotation as a function of radial position within the cell. They found that with increased shear strain, the rate of rotation of the director within the cell slows down. These observations are all consistent with a director that remains within the shear plane during shearing, but is slowed by an increasing elastic component to the shear stress due to windup of the director from the top plate to the bottom plate. Mather et al. also found that the damped stress oscillations were completely reversible at short strains $(\gamma \approx 200)$, but irreversible at longer strains, just as in the cone and plate experiments of $\mathrm{Gu}$ and Jamieson. The optical experiments revealed a proliferation of disclinations at higher strains and Mather reasoned that this creation of disclinations is ultimately responsible for the lack of reversibility. As a result of their work in the parallel disk geometry, these authors suggested that a similar mechanism involving increasing in-plane elastic wind-up; and the onset of disclinations, may also be responsible for the damped stress oscillations and irreversibility at higher strains observed in the cone-plate geometry, though, of course, without appreciable phase mixing.

Unfortunately, however, a careful review of the earlier literature suggests that the Mather et al. explanation for damping of the oscillatory stress upon startup of shear may not apply to flow systems other than the parallel disk (or torsional) flow. In particular, published experimental and theoretical work seems to provide a strong inference that the dominant mechanism depends on the geometry of the flow.

Experimentally, the occurrence of in-plane and outof-plane instabilities has been studied extensively by the groups of Cladis and Torza (1975, 1976), Pieranski (Pieranski and Guyon 1974, 1976; Pieranski et al. 1976), and Carlsson (Skarp et al. 1981; Carlsson 1984; Carlsson and Skarp 1986). What is meant by an in-plane instability is one in which the director remains within the shear plane but undergoes a rotation through the flow direction (commonly called tumbling). An out-of-plane instability refers to the transition where the director takes on components of orientation in the vorticitydirection, out of the plane of shear.

Pieranski's group, working with the tumbling nematic HBAB in the parallel plate geometry (simple shear flow) with the director initially in the planar configuration (in shear plane parallel to flow direction), found that, when gradually increasing the shear-rate, a certain shear-rate is reached at which the director turns out of the shear plane. At larger shear-rates the director is nearly perpendicular to the plane of shear and with an even further increase of shear-rate, a cellular instability (roll cells) was observed. This behavior is fundamentally different from that observed by Mather et al. in the parallel disk (torsional) flow.

Cladis and Torza $(1975,1976)$ studied cylindrical Couette flow of the tumbling nematics HBAB and CBOOA. With homeotropic orientation at the walls (i.e., director in the shear plane perpendicular to the walls), the shear-rate was increased gradually from zero. At low shear-rates, the first instability observed in this case was an in-plane tumbling instability. This was followed, at higher shear-rates, by a rotation of the director out of the shear plane in the regions near the boundaries, but with the director elsewhere remaining largely within the plane of shear. With continued shearing, bands of disclinations were seen to originate from these highly twisted regions that developed near the boundaries. Finally, with a further increase of shear-rate they report a second instability to a roll-cell regime very much like that reported by Pieranski's group. In contrast to the work of Pieranski, however, Cladis and Torza report a stable regime after the first instability in which the director remains in the shear plane throughout most of the gap. 
Finally, Carlsson's group studied the tumbling instability in torsional flow (parallel disk) starting from a homeotropic monodomain with the tumbling nematic $8 \mathrm{CB}$. In their work, they found that the out-of-plane instability does not occur in torsional flow. In this particular geometry the director is thought to be stabilized against fluctuations out of the shear plane by a hydrodynamic "back flow" (Chandrasekhar 1992). This is consistent with the work of Mather et al. (1997), with the exception of the very thin twist-wall regions in which the director twists out of the shear plane into the vorticity direction and then returns to the shear plane.

So, from the existing experimental work, it appears that the onset of these in-plane and out-of-plane instabilities is dependent on the particular geometry of the flow and possibly the initial orientation, planar or homeotropic.

The initial theoretical work on hydrodynamic instabilities in liquid crystals dealt with the simplified case of simple shear flow with the director assumed to remain within the shear plane. By numerical integration of the 2D LE equations for simple shear flow, Manneville (1981) found that the maximum tilt angle of the director, $\theta_{\mathrm{m}}$, is a multi-valued function of the shearing velocity $\left(\theta_{\mathrm{m}}\right.$ is the angle measured from velocity gradient direction, $\nabla v$ ). For a tumbling nematic, at very small velocities the director at the midplane will rotate through a small angle, $\theta_{\mathrm{m}}<\pi / 2$, with the viscous forces being balanced by the elastic stress. As the velocity increases, the viscous force becomes large enough that the director is able to rotate to a new configuration in which $\pi / 2<\theta_{\mathrm{m}}<\pi$. Carlsson (1984) also numerically integrated the 2D LE equations for simple shear flow using the one constant simplification $\left(K_{1}=K_{3}\right)$ and found that the tilt angle at the midplane, $\theta_{\mathrm{m}}$, is multivalued only for small negative values of $\alpha_{3} / \alpha_{2}$ (low $\lambda$ ).

Realizing that at some point the director may become unstable to rotations out of the shear plane, the more recent work has considered the full 3D LE continuum equations. Pieranski et al. (1976) considered simplified equations valid only for small distortions about planar orientation and reported two instabilities: one in the shear plane and the other out of the plane of shear with the corresponding thresholds (shearing velocities, $V$ ) for each so close to each other that the former is concealed by the latter. Zúñiga and Leslie (1989a, b) report similar results for the case of simple shear flow. In the first paper (Zúñiga and Leslie 1989a), they use the one constant approximation $\left(K_{1}=K_{2}=K_{3}\right)$ and show that although the first instability is in-plane, the director tumbles to a new configuration which is unstable to out-of-plane rotations, while for some cases with homeotropic boundary conditions the first instability is in-plane but again the director tumbles to an orientation which is immediately unstable to out-of-plane rotations. Zúñiga (1990) later considered cylindrical Couette flow using the full equations without the one constant approximation and reports that the rotation and cylindrical geometry have a slight stabilizing effect in that the director achieves a bigger angle, $\theta_{\mathrm{m}}$, before becoming unstable. The first instability is still out-of-plane for all but the lowest temperatures (low $\lambda$ ). The low $\lambda$ solution set has an in-plane instability first, then tumbles to a $\theta_{\mathrm{m}}$ which is unstable to out-of-plane rotations of the director. Also, the solution after the out-of-plane instability is stable again to out-of-plane rotations. So it is possible, if the director somehow returns to the shear plane after the out-of-plane instability, for the director to undergo multiple in-plane rotations.

We should note that there is an important difference between the theoretical works by Manneville, Carlsson, Pieranski, and Zúñiga, in that the simulations approach the instability problem by increasing $E r$ gradually from zero and looking for the first instability to appear, inplane or out-of-plane. In our experiments, we start the flow at an $E r$ which is already much greater than that required for an instability and seek to determine which instability takes place first, in-plane or out-of-plane.

Han and Rey (1993) took the analysis a step further by determining the steady-state director fields (following the trajectories after the out-of-plane instability) for simple shear flow for both planar and homeotropic boundary conditions. They found both in-plane and out-of-plane solutions with the steady state director pattern dependent on the initial director orientation and the magnitude of the Ericksen number. In later papers Han and Rey (1994a, b, 1995) use an adaptive numerical method to solve the full non-planar LE equations and determine the trajectories of the director on flow startup. Although their work was only on simple shear flow, their results show that director profiles may be obtained which match those observed by Pieranski's group (director gradually twisting further out-of-plane with increasing strain) as well as those of Cladis (regions outof-plane only near boundaries with the director in the bulk region remaining within the shear plane) by simply altering the initial condition from planar to homeotropic. Han and Rey's results however do not adequately predict the stress damping or the reversibility observed in the cone-plate work of $\mathrm{Gu}$ et al. (1993) possibly due to the Ericksen number gradient present in the coneplate geometry or the inability to account for disclinations in their simulations.

What is interesting about the theoretical work is the influence of the elastic constants on the out-of-plane instability. By removing the one constant approximation $\left(K_{1}=K_{2}=K_{3}\right)$, Zúñiga (1990) was able to find solutions with multiple in-plane instabilities before the director eventually becomes unstable to an out-of-plane instability. Han and Rey $(1994 b, 1995)$ suggest that the outof-plane instability is favored as a means of replacing the relatively costly bend-splay distortions of the director in 
the shear plane with a twist distortion of the director out of the shear plane. Also, the dependence on geometry and initial conditions (planar or homeotropic) makes sense when thought about in this way since different geometries or initial conditions might favor some elastic modes more than other ones.

Our goal in this work is to better understand the mechanism responsible for the stress oscillation damping observed in the cone-plate cell. We believe that this mechanism is related to the point at which the director becomes susceptible to out-of-plane rotations of the director. So our approach is to examine more closely the start-up flow of a tumbling nematic in two different geometries: the cylindrical Couette cell and the coneplate cell. First, we look at the stress oscillations upon flow start-up in the cylindrical Couette cell. Two reasons make this particular flow cell an obvious place to start. One, extensive optical experiments have already been conducted by Cladis' group and two, the flow is uniform in shear-rate and Ericksen number, making it free of some of the complications present in the parallel disk $(\nabla \dot{\gamma})$ and cone-plate geometries $(\nabla E r)$. By adding our new rheological measurements to the published optical experiments in this flow cell we are able to propose transient director profiles which are consistent with all the experimental evidence. Next, we conduct new optical experiments on a tumbling nematic in the cone-plate cell and hope to apply what we've learned from the cylindrical Couette case to propose an explanation for the mysterious damping and irreversibility phenomena in this geometry.

\section{Experimental methods}

\section{Materials}

Here we study the small molecule LC, 4'-n-octyl-4-cyanobiphenyl (8CB). This LC was obtained from EM Industries and used as received. The phase transition temperatures reported for $8 \mathrm{CB}$ are $20.5,33.3$, and $40.1{ }^{\circ} \mathrm{C}$ for the crystalline-smectic-A, smectic-Anematic, and nematic-isotropic transitions respectively (Karat and Madhusudana 1976).

\section{Techniques}

The stress oscillation experiments have been performed and explained previously by Gu et al. (1993) and Ternet et al. (1999), so only a brief description of the technique will be given here. The experiments here were carried out with the ARES rheometer using the cylindrical Couette and cone-plate geometries. The cylindrical Couette cell had a cup of diameter $34 \mathrm{~mm}$ and three different custom bobs were used, one with a length of $33 \mathrm{~mm}$ and a diameter of $32 \mathrm{~mm}$ and the others with a length of $29 \mathrm{~mm}$ and diameters of $33 \mathrm{~mm}$ and $33.5 \mathrm{~mm}$ giving gaps of $1.0 \mathrm{~mm}, 0.5 \mathrm{~mm}$, and $0.25 \mathrm{~mm}$, respectively. The cone-plate cell had a diameter of $50 \mathrm{~mm}$ and a cone angle of 0.04 radians. Initially, the shearing surfaces of the flow cell were treated with a dilute solution of lecithin in chloroform to achieve a uniform homeotropic monodomain. The flow was then started from rest and the transient shear stress measured.
Optical experiments were carried out in a custom cone-plate flow cell made with fused silica cone and plate fixtures for transparency. The cone had an angle of 0.04 radians to match that of the ARES rheometer. Again, the surfaces were treated with lecithin to obtain a homeotropic monodomain. The flow was started from rest and observed under crossed polarizers with the polarizer in the flow direction and the analyzer in the vorticity direction. A more detailed description of the technique is given by Mather et al. (1997).

In our experiments, we only look at the homeotropic initial condition mainly because of the ease with which it is obtained relative to that of the planar boundary condition. A planar boundary condition is typically obtained by applying a rubbed polymer coating to the surfaces, which is particularly difficult to apply in the cone-plate geometry. Also, the experiments of Cladis and Torza $(1975,1976)$ with the cylindrical Couette cell were obtained with a homeotropic initial condition and we intend to make comparisons with their work by using the same initial condition.

\section{Results and discussion}

\section{Cylindrical Couette experiments}

We start by presenting our results for the cylindrical Couette cell. Unlike the parallel disk or cone-plate cells, the Couette cell has both a uniform gap and shear-rate, so that $E r$ is constant throughout the cell. As a result, we expect it to be easier to get to the cause of any damping observed in this geometry. No optical experiments were possible in this particular flow cell so we will rely on the observations published by Cladis and Torza $(1975,1976)$ when presenting an explanation for the behavior of the tumbling nematic in the cylindrical Couette cell. Prior to our work, there existed no shear stress data for a tumbling nematic in the cylindrical Couette cell, so the evolution of the director patterns first proposed by Cladis and Torza was only supported by optical observations.

The experiments were carried out using the tumbling nematic $8 \mathrm{CB}$ in the cylindrical Couette cell of the ARES rheometer. Our initial work using the cylindrical Couette cell was done with the 32-mm bob giving the cell a gap of $1 \mathrm{~mm}$. Transient shear start-up runs were conducted at temperatures ranging from $33.7^{\circ} \mathrm{C}$ to $41.5^{\circ} \mathrm{C}$, at shearrates ranging from $1 \mathrm{~s}^{-1}$ to $256 \mathrm{~s}^{-1}$. Rather than reproduce the results from this entire range here, we only discuss the interesting points and present figures of the actual data when necessary. For temperatures below $36.0{ }^{\circ} \mathrm{C}$, we see immediate damping of stress oscillations at all shear-rates studied, similar to the results obtained with the cone-plate geometry ( $\mathrm{Gu}$ et al. 1993). For temperatures between $36.0^{\circ} \mathrm{C}$ and $38.0{ }^{\circ} \mathrm{C}$ we see damping immediately from the first overshoot at lower shear-rates, while at shear-rates greater than $16 \mathrm{~s}^{-1}$ we find that damping begins only after the second or third overshoot, indicating that the first 1-2 rotations of the director remain within the shear plane. At temperatures above $38.0^{\circ} \mathrm{C}$, immediate stress damping was observed 
at all shear-rates. An example of the initially damped and undamped transient stress oscillations seen in each of the three temperature ranges $\left(\mathrm{T}<36{ }^{\circ} \mathrm{C}, 36^{\circ} \mathrm{C} \leq \mathrm{T} \leq 38{ }^{\circ} \mathrm{C}\right.$, $\left.38^{\circ} \mathrm{C}<\mathrm{T}\right)$ is shown in Fig. 2.

After the initial set of runs with the $32 \mathrm{~mm}$ diameter bob, we ran the same experiments with the two larger bobs with diameter $33 \mathrm{~mm}$ and $33.5 \mathrm{~mm}$ at a single temperature, $36.8^{\circ} \mathrm{C}$. Use of these bobs allows us to vary the gap and hence vary the Ericksen number and test for the effect of elasticity on the transition from inplane to out-of-plane rotations of the director. The results for all three bobs are consistent with the idea that a critical Ericksen number, $E r=\eta \dot{\gamma} h^{2} / K$, controls the inplane and out-of-plane instabilities. With the $32 \mathrm{~mm}$ diameter bob $(h=1 \mathrm{~mm})$, in the temperature range of $36.0{ }^{\circ} \mathrm{C}$ to $38.0{ }^{\circ} \mathrm{C}$, at the lower shear-rates the transient stress begins to dampen immediately after the first overshoot while at shear-rates greater than $16 \mathrm{~s}^{-1}$, the stress begins to dampen after the second or third oscillation peak. With the $33 \mathrm{~mm}$ diameter bob $(h=0.5 \mathrm{~mm})$, at a temperature of $36.8{ }^{\circ} \mathrm{C}$, the stress dampens immediately for all shear-rates less than around $64 \mathrm{~s}^{-1}$, while those greater than $64 \mathrm{~s}^{-1}$ begin to dampen after the second or third overshoot. With the $33.5 \mathrm{~mm}$ diameter bob $(h=0.25 \mathrm{~mm})$, at a temperature of $36.8{ }^{\circ} \mathrm{C}$, the stress dampens immediately for all shear-

Fig. 2 Transient shear stress obtained with $8 \mathrm{CB}$ when starting with a homeotropic monodomain in cylindrical Couette cell with $32-\mathrm{mm}$ bob at $32 \mathrm{~s}^{-1}$ at temperatures of $35.5^{\circ} \mathrm{C}, 37.0^{\circ} \mathrm{C}$, and $38.5^{\circ} \mathrm{C}$. (Note: curves are shifted for clarity) rates studied up to $256 \mathrm{~s}^{-1}$. It is difficult to obtain reliable data at higher shear-rates due to the limited time resolution of the ARES rheometer. Based on the data for the three different bobs giving gaps of $0.25 \mathrm{~mm}$, $0.5 \mathrm{~mm}$, and $1.0 \mathrm{~mm}$, it is clear that as the gap doubles the critical shear-rate for undamped stress oscillations goes up by a factor of 4 consistent with the idea that a critical Ericksen number is determining the transition to out-of-plane tumbling. If we use typical values for the ratio $\eta / K$ of $6 \times 10^{5} \mathrm{~cm}^{2} / \mathrm{sec}$ (Kneppe et al. 1982; Karat and Madhusudana 1976), we can estimate the critical Ericksen number to be between 100,000 and 200,000.

Reversibility studies were also conducted with the $32 \mathrm{~mm}$ diameter bob at a temperature of $36.8^{\circ} \mathrm{C}$. The results were very similar to those obtained and presented earlier with the cone-plate geometry. If the shearing is reversed at short strains ( $\gamma \approx 100$ strain units), the stress damping is completely reversible and the damped stress oscillations return with growing amplitude. If the shearing is allowed to continue to higher strains $(\gamma \approx 300$ strain units), the transient stress is no longer reversible and the stress oscillations do not return.

Finally, we note that this immediate damping at higher shear rates observed in the temperature ranges below $36.0{ }^{\circ} \mathrm{C}$ and above $38.0{ }^{\circ} \mathrm{C}$ might simply be an experimental artifact. This range of temperature between $36.0^{\circ} \mathrm{C}$ and $38.0{ }^{\circ} \mathrm{C}$ happens to correspond to the range of temperatures at which the curve for $\lambda$ vs temperature for $8 \mathrm{CB}$ levels out and becomes very flat as shown in Fig. 3. This may indicate that the damping observed at other temperatures in the Couette cell is just

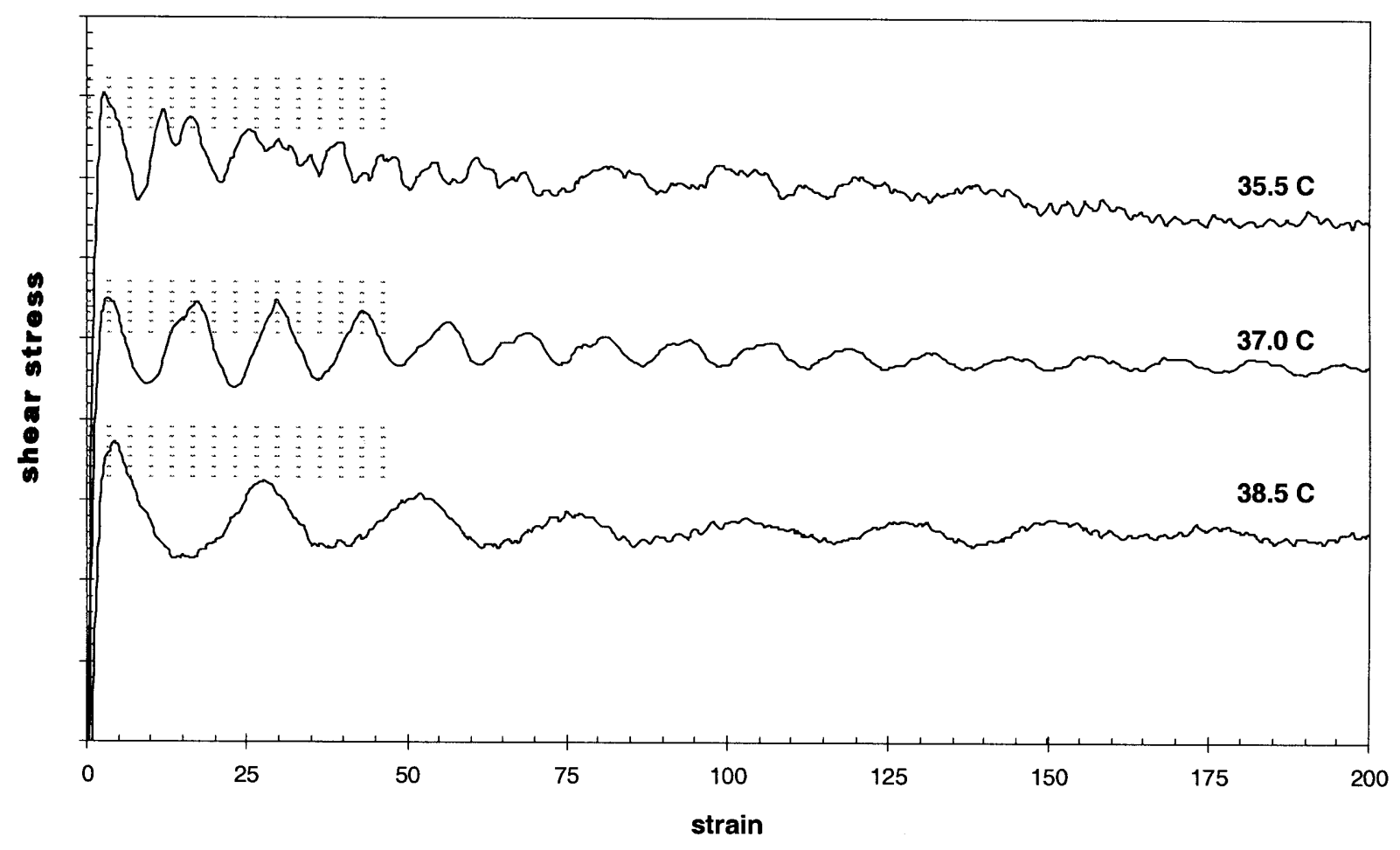




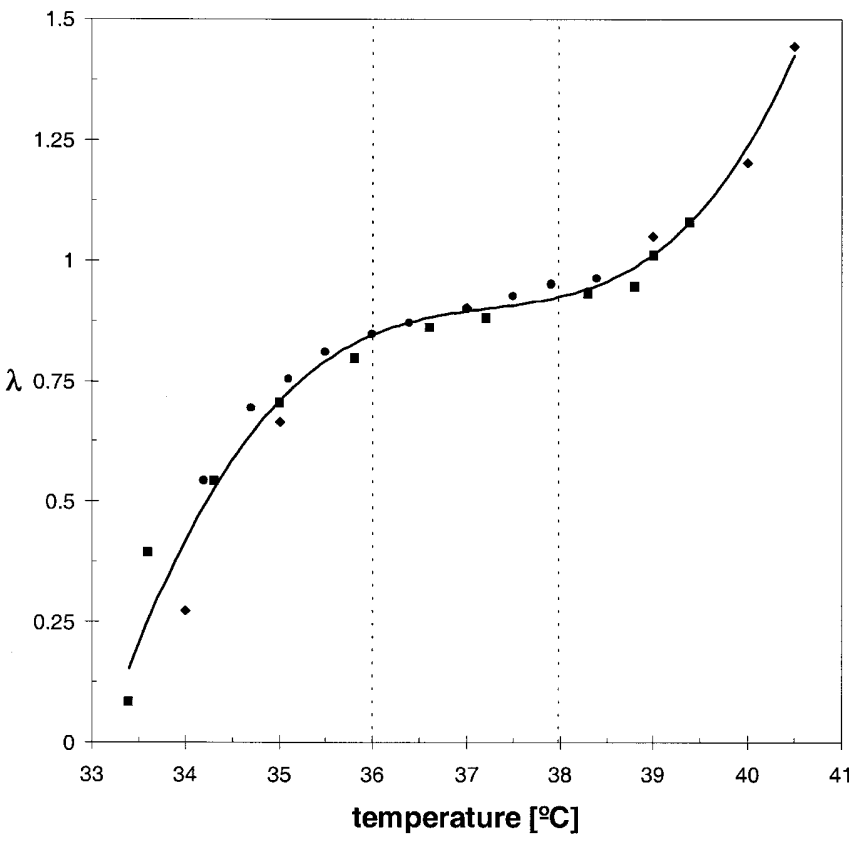

Fig. $3 \lambda$ vs T for 8CB as determined by Ternet et al. (1999) (O), Gu and Jamieson (1994) (ם), and Kneppe et al. $(1981,1982)$

due to a slight temperature gradient within the cell. A slight temperature change would lead to a larger $\Delta \lambda$ within the cell and hence faster or slower tumbling leading to phase-mixing which would dampen the oscillations. A check of the temperature within the cell revealed a variation of at most $0.2{ }^{\circ} \mathrm{C}$, which although small may be enough to cause some phase-mixing at temperatures where $\lambda$ is changing rapidly with temperature such as those below $36.0^{\circ} \mathrm{C}$ and above $38.0^{\circ} \mathrm{C}$ in $8 \mathrm{CB}$.

Fig. 4 Schematic depicting proposed transient director patterns upon flow start-up of tumbling nematic in cylindrical Couette cell
Proposed start-up director patterns for the cylindrical Couette cell

Here we present an explanation for the damped stress oscillations in the simple geometry of the cylindrical Couette cell which is consistent with our experimental observations and those of Cladis and Torza as well as the simulations of Han and Rey. Cladis and Torza $(1975,1976)$ observed the steady state director patterns in the Couette cell, as the shear-rate $(E r)$ was increased slowly from zero. Han and Rey (1994a, b, 1995) have shown that such patterns may also develop in simple shear flow between parallel plates with homeotropic boundary conditions. In Han and Rey's simulations, as the shearing begins, the director initially rotates in-plane and builds up elastic energy due to splay-bend distortions of the director field from the top plate to the bottom plate. At higher strains, the director rotates outof-plane in regions near the boundaries, replacing the costly splay-bend distortions with a twist distortion. The director in the bulk remains within the plane of shear, in agreement with the observations of Cladis and Torza. At even higher strains, the twist distortions near the boundary occur over smaller and smaller distances (like the continued winding of a spring). Although not predicted by the simulations, we can guess that as the director field becomes more and more distorted in these boundary regions, disclinations will develop out of these regions similar to those observed by Cladis and Torza.

Our proposed progression of director patterns in the Couette cell upon start-up of shear is depicted in Fig. 4. As flow begins, the director remains within the shear plane and rotates through the first in-plane tumbling instability (A,B in Fig. 4). After the first in-plane instability and before a second in-plane instability, the director becomes unstable to rotations out of the shear

\section{(A)}

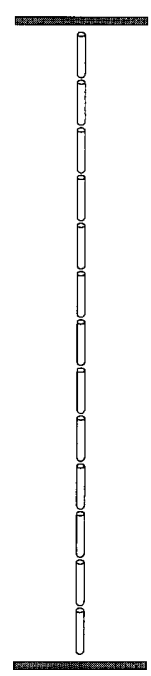

\section{(B)}

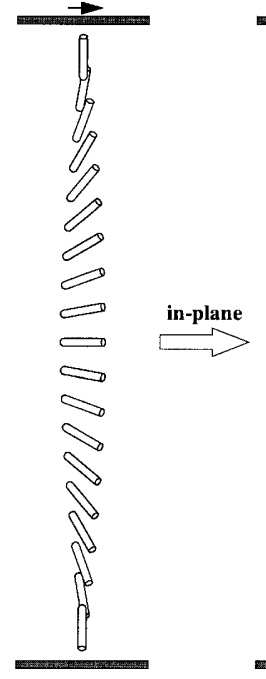

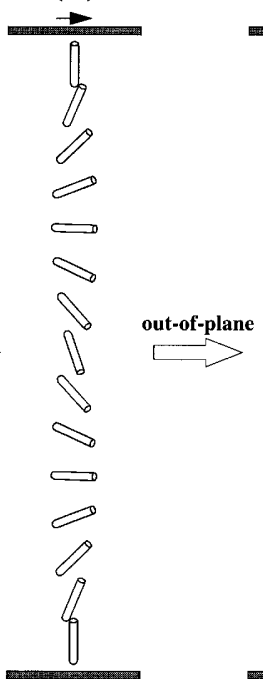

(C)

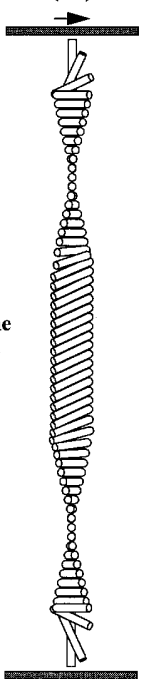

(D)

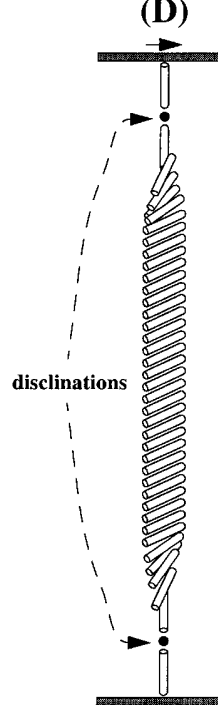


plane. The director then tumbles out of the shear plane and the stress begins to dampen (B,C in Fig. 4). As the out-of-plane rotations continue, the regions near the boundaries develop greater and greater twist distortions, with the bulk region remaining within the shear plane. Eventually as the director field becomes more and more distorted, disclinations form and begin to nucleate from these highly twisted boundary regions (C,D in Fig. 4). The disclinations shown in Fig. 4 are not meant to represent the actual structure of the disclinations, but rather to indicate regions where disclinations are forming. After these disclinations form, the stress damping becomes irreversible as the director field is no longer continuous from the inner cylinder to the outer. A comparison between the evolution of the apparent viscosity and the proposed director patterns of Fig. 4 may be seen in Fig. 5, where the regions A, B, C, and D are labeled. This proposed progression of transient director patterns is consistent with our stress measurements as well as the optical experiments of Cladis and Torza. Also, patterns similar to these have been predicted by Han and Rey (1994a, b).

\section{Cone and plate experiments}

After obtaining an explanation for the stress damping in the less complicated geometry of the cylindrical Couette cell, we now present new optical experiments on the cone-plate cell to try to explain the damping first observed by Gu et al. (1993) in this geometry. In these optical experiments we hoped to find distinct twist wall
Fig. 5 Response of tumbling nematic in cylindrical Couette cell for flow reversal at: top: short strains; bottom: long strains, with regions $\mathrm{A}, \mathrm{B}, \mathrm{C}$, and $\mathrm{D}$, from Fig. 4 show
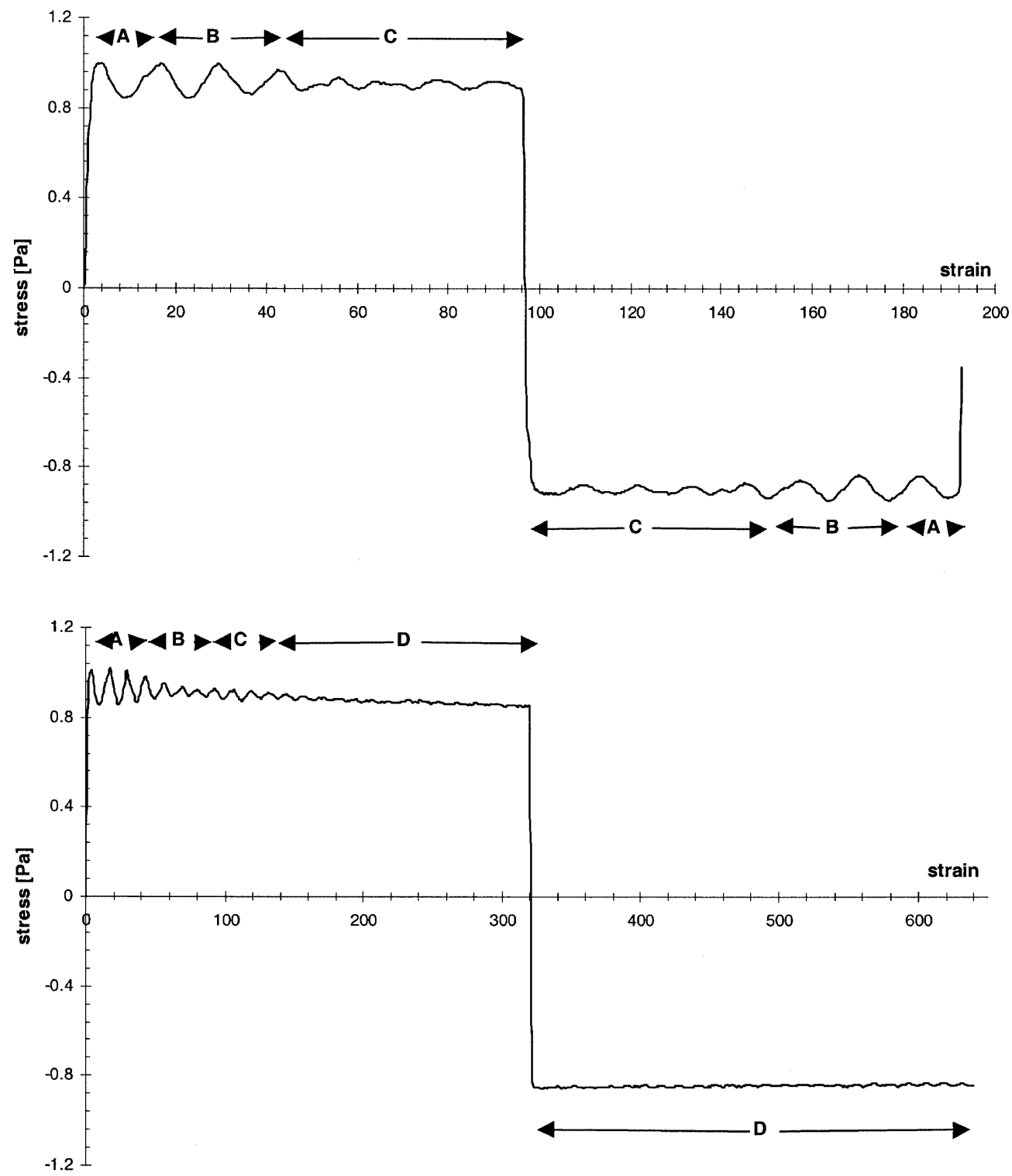
regions like those observed by Mather et al. (1997) in the parallel disk cell which could be used to track the rate of rotation of the director within the cone-plate cell. With the polarizer in the flow direction and the analyzer oriented in the vorticity direction, the flow was started from rest at shear-rates ranging from $0.25 \mathrm{~s}^{-1}$ to $125 \mathrm{~s}^{-1}$. The experiments were conducted at $35.5^{\circ} \mathrm{C}, 36.5^{\circ} \mathrm{C}$, and $37.0^{\circ} \mathrm{C}$. At these temperatures, the corresponding tumbling parameters for $8 \mathrm{CB}$ were $\lambda \approx 0.81, \lambda \approx 0.87$, and $\lambda \approx 0.90$, respectively (Ternet et al. 1999).

Unlike the parallel disk results of Mather et al. (1997), we observed no twist-walls upon start-up of flow for any of the shear-rates and temperatures studied. Instead, we see a progression of textures leading to a disclination filled sample of varying brightness. These results would indicate that the particular instability leading to the twist-walls in the parallel disk geometry is specific to that particular geometry. It appears that in the cone-plate geometry, the director either never rotates out-of-plane or does so uniformly throughout the cell rather than being limited to localized regions as was observed in the parallel disk flow cell. So, without the twist walls to allow us to track the rate of rotation of the director at different radial positions within the cell, we are unable to say whether the rate of rotation of the director is slowed by an elastic effect. Conoscopy experiments would provide better information on whether the director is tipping out-of-plane immediately from start-up.

However, we can use what we've learned from our experiments with the cylindrical Couette cell to guess at a possible explanation for the cone-plate cell. As we found in the cylindrical Couette cell, there is a critical Ericksen number below which the director is immediately unstable to out-of-plane tumbling. A similar critical Ericksen number might also exist for the cone-plate cell. Due to the gradient in $\mathrm{Er}$ from the center of the cone to the outer edge, there will always be some region near the center with an $E r$ below the critical Er. Even though the contribution to the overall torque from the center region in the cone-plate cell is much less than that at the edge it may still be enough to decrease appreciably the magnitude of the second overshoot. Also, the out-of-plane tipping at the center of the cone-plate cell may trigger further out-of-plane tipping in regions near the edge of the cell even though the Ericksen number there is above the critical $E r$. The director patterns might be similar to those we proposed for the cylindrical Couette cell including the bands of disclinations forming near the boundary regions which would explain the lack of reversibility seen at larger strains.

\section{Conclusion}

In this work, our intent was to determine the origin of stress oscillation damping observed in the cone-plate flow cell. To do so the simpler geometry of the cylindrical Couette cell was examined more closely. The transient stress upon flow start-up in the cylindrical Couette cell revealed some conditions $\left(36.0^{\circ} \mathrm{C} \leq \mathrm{T} \leq\right.$ $38.0^{\circ} \mathrm{C}, 32 \mathrm{~s}^{-1}=\dot{\gamma}$ ) in which the stress damping begins after the second or third oscillation peak. Under these conditions it is presumed that the initial 1-2 rotations of the director remain in-plane, after which the director rotates out-of-plane and the stress oscillations dampen. We also found evidence for a critical Ericksen number controlling the position of the first out-of-plane instability. Reversibility studies revealed a critical strain beyond which the transient stress oscillations do not return if the flow is reversed. Combined with the observations of Cladis and Torza of bands of disclinations developing in highly twisted regions near the boundaries at higher strains, we propose that the nucleation of disclinations effectively breaks continuity of the director from one plate to the other and spoils the reversibility. The immediate stress oscillation damping observed in the cone-plate cell suggests that in this geometry the director is immediately unstable to out-ofplane rotations of the director. Similar to the case of the cylindrical Couette cell, we expect that the nucleation of disclinations is responsible for the lack of reversibility of the transient stress at longer strains.

Incorporating Frank elasticity into the Doi model for LCPs has received a great deal of attention lately (Marrucci and Greco 1992; Feng et al. 2000). The initial work has been concentrated on 2D-channel flow and later work will undoubtedly need to consider the 3D case. It is hoped that the insights gained from the current work (role of elasticity, geometry, and initial conditions) with these much simpler small-molecule LC systems will prove helpful.

\section{References}

Burghardt WR, Fuller GG (1990) Transient shear flow of nematic liquid crystals: manifestations of director tumbling. J Rheol 34:959-992

Carlsson T (1984) Theoretical investigation of the shear flow of nematic liquid crystals with the Leslie viscosity $\alpha_{3}>0$ : hydrodynamic analogue of first order phase transitions. Mol Cryst Liq Cryst 104:307-334

Carlsson T, Skarp K (1986) Observation of the tumbling instability in torsional shear flow of a nematic liquid crystal with $\alpha_{3}>0$. Liq Cryst 1:455-471
Chandrasekhar S (1992) Liquid crystals. Cambridge University Press

Cladis PE, Torza S (1975) Stability of nematic liquid crystals in Couette flow. Phys Rev Lett 35:1283-1286 
Cladis PE, Torza S (1976) Colloid and interface science, vol IV. Academic Press, pp 487-499

Ericksen JL (1960) Anisotropic fluids. Arch Ration Mech Anal 4:231-237

Feng JJ, Sgalari G, Leal LG (2000) A theory for flowing nematic polymers with orientational distortion. J Rheol 44:1085-1101

Gu D-F, Jamieson AM (1994) Shear deformation of homeotropic monodomains: temperature dependence of stress response for flow-aligning and tumbling nematics. J Rheol 38:555-571

Gu D-F, Jamieson AM, Wang S-Q (1993) Rheological characterization of director tumbling induced in a flow-aligning nematic solvent by dissolution of a sidechain liquid-crystal polymer. J Rheol 37:985-1001

Han WH, Rey AD (1993) Supercritical bifurcations in simple shear flow of a non-aligning nematic: reactive parameter and director anchoring effects. J NonNewtonian Fluid Mech 48:181-210

Han WH, Rey AD (1994a) Dynamic simulations of shear flow induced chirality and twisted texture transitions of a liquid crystalline polymer. Phys Rev E 49:597-614

Han WH, Rey AD (1994b) Simulation and validation of nonplanar nematorheology. J Rheol 38:1317-1334
Han WH, Rey AD (1995) Simulation and validation of temperature effects on the nematorheology of aligning and nonaligning liquid crystals. J Rheol 39:301-322

Karat PP, Madhusudana NV (1976) Elastic and optical properties of some $4^{\prime}-n$ alkyl-4-cyanobiphenyls. Mol Cryst Liq Cryst 36:51-64

Kneppe H, Schneider F, Sharma NK (1981) A comparative study of the viscosity coefficients of some nematic liquid crystals. Ber Bunsenges Phys Chem 85:784-789

Kneppe H, Schneider F, Sharma NK (1982) Rotational viscosity $\gamma_{1}$ of nematic liquid crystals. J Chem Phys 77:3203-3208

Manneville P (1981) The transition to turbulence in nematic liquid crystals. Mol Cryst Liq Cryst 70:223-250

Marrucci G, Greco F (1992) A molecular approach to the polydomain structure of LCPs in weak shear flows. J NonNewtonian Fluid Mech 44:1-13

Mather PT, Pearson DS, Larson RG, Gu D-F, Jamieson AM (1997) The origin of stress-oscillation damping during start-up and reversal of torsional shearing of nematics. Rheol Acta 36:485-497

Pieranski P, Guyon E (1974) Two shearflow regimes in nematic $p$ - $n$-hexyloxybenzilidene- $p^{\prime}$-aminobenzonitrile. Physical Review Letters 32:924-926
Pieranski P, Guyon E (1976) Shear flow instabilities in nematic CBOOA. Commun Phys 1:45-49

Pieranski P, Guyon E, Pikin SA (1976) Novelles instabilités de cisaillement dans les nématiques. J Phys Colloq 37: $\mathrm{C} 1-3$

Skarp K, Carlsson T, Lagerwall ST, Stebler B (1981) Flow properties of nematic 8CB: an example of diverging and vanishing $\alpha_{3}$. Mol Cryst Liq Cryst 66:199-208

Ternet DJ, Leal LG, Larson RG (1999) Flow-aligning and tumbling in smallmolecule liquid crystals: pure components and mixtures. Rheol Acta 38:183-197

Zúñiga I (1990) Orientational instabilities in Couette flow of non-flow-aligning nematic liquid crystals. Phys Rev A 41:2050-2058

Zúñiga I, Leslie FM (1989a) Shear flow instabilities in nonaligning nematic liquid crystals. Europhys Lett 9:689-693

Zúñiga I, Leslie FM (1989b) Shear flow instabilities in non-flow-aligning liquid crystals. Liq Cryst 5:725-734 